\title{
Emotional Intelligence as Predictor of Mental Health among Chronic Disease Group
}

\author{
$\operatorname{Manju}^{1 *}$
}

\section{ABSTRACT}

Health is considered a sense of wellbeing, inner happiness, and enthusiasm for living and harmony within self and with others, absence of disorders, conflicts, worries and anxieties. Emotional reactions and experiences affect both physical as well as psychological health. Negative emotional states are associated with unhealthy patterns of physiological functioning, whereas positive emotional states are associated with healthier patterns of responding in both cardiovascular activity and immune system. Taylor (2001) argues that emotionally intelligent people can cope better with life's challenges and control their emotions more effectively, both of which contribute to good psychological and physical health. So the present study was designed to see the relationship between emotional intelligence and mental health and to see the role of emotional intelligence in mental health. Multidimensional Measure of Emotional Intelligence (MMEI by C. R. Darolia, 2003) and General Health Questionnaire (GHQ by Goldberg \& Williams, 1988) was used. Sample of the study comprised of 200 chronic patients. Data obtained was analyzed by using co-relation and regression analysis. Results revealed emotional intelligence is positively correlated with mental health and emerged as predictor of mental health.

Keywords: Emotional Intelligence, Predictor, Mental Health, Chronic Disease Group

Salovey and Mayer (1990) coined the term 'emotional intelligence' and described it as a form of social intelligence that involves the ability to monitor one's own and others feelings and emotions to discriminate among them and to use this information to guide one's thinking and action. Mayer and Salovey (1997) elaborated that emotional intelligence as 'the ability to perceive emotions, to access and generate emotions so as to assist thought, to understand emotions and emotional knowledge, and to reflectively regulate emotions so as to promote emotional and intellectual growth'. Emotional intelligence according to them involves areas such as 'identifying emotions; the ability to recognize how you and those around you are feeling using

\footnotetext{
${ }^{1}$ Assistant professor, Department of applied psychology, GJUS \& T, Hisar, India *Responding Author

(C) 2016 I Manju; licensee IJIP. This is an Open Access Research distributed under the terms of the Creative Commons Attribution License (http://creativecommons.org/licenses/by/2.0), which permits unrestricted use, distribution, and reproduction in any Medium, provided the original work is properly cited.
} 


\section{Emotional Intelligence as Predictor of Mental Health among Chronic Disease Group}

emotions - the ability to generate emotional and then reason with this emotion, understanding emotions - the ability to understand complex, emotions and emotional 'chains', how emotions transition from one stage to another, and managing emotions - the ability which allows you to manage emotions in yourself and in others. Goleman (1995) made a provocative claim that it Intelligence Quotient contribute up to $25 \%$ to life's success, the remaining was fulfilled through one's emotional intelligence and as a result predicted "Emotional Intelligence would contribute to the success at home, at school and at work.”

Mayer and Salovey (1990) defined emotional intelligence as "the ability to monitor one's own and others' feelings and emotions, to discriminate among them and to use this information to guide one's thinking and actions."

Individuals who can regulate their emotions are healthier because they 'accurately perceive and appraise their emotional states, know how and when to express their feelings, and can effectively regulate their mood states'. This set of characteristics, dealing with the perception, expression, and regulation of moods and emotions, suggests that there must be a direct link between emotional Intelligence and physical as well as psychological health (Salovey et al., 1999). An emotionally intelligent person can cope better with life's challenges and control their emotions more effectively, both of which contribute to good psychological and physical health (Taylor, 2001). There is a relationship between emotional Intelligence, stress and a number of measures of psychological health, such as depression, hopelessness and suicidal ideation among young people (Ciarrochi, Deane \& Anderson, 2002).

Lack of emotional awareness and inability to manage emotions are key symptoms in some personality disorders and impulse control disorders (Matthews et al., 2002). Lower emotional intelligence and lack of awareness of emotional processes as well as impulse control problems are linked together; lower emotional intelligence is also associated with more alexithymia and less impulse control (Schutte et al., 1998).

Matthews et al. (2002) pointed out that medical disorders, especially ones with psychosomatic aspects, are often co-morbid with mood or anxiety disorders. Higher emotional intelligence is linked with aspects of better psychosocial functioning (e.g., Schutte et al., 1998; Schutte et al., 2001; Salovey \& Grewal, 2005; Brown \& Schutte, 2006), including intrapersonal factors such as greater optimism and interpersonal factors such as better social relationships. Some of these psychosocial factors, such as more social support and more satisfaction with social support for those with higher emotional intelligence (Brown \& Schutte, 2006), may serve as buffers to physical illness. Further, those with higher emotional intelligence might be better able to follow through on commitments to health behavior and show better medical compliance. 


\section{Emotional Intelligence as Predictor of Mental Health among Chronic Disease Group}

Emotional reactions and experiences affect both physical as well as psychological health. Negative emotional states are associated with unhealthy patterns of physiological functioning, whereas positive emotional states are associated with healthier patterns of responding in both cardiovascular activity and immune system (Booth-Kewley \& Friedman, 1987; Herbert \& Choen, 1993). Salovey et al. (2000) discussed extensively the significance of emotional states on physical health suggesting that an individual's emotional status influence their perception of physical symptoms. This set of characteristics, dealing with the perception, expression, and regulation of moods and emotions, suggests that there must be a direct link between emotional intelligence and physical as well as psychological health. By keeping the above review in mind the present study was designed to see the relationship between emotional intelligence and mental health.

\section{Objectives}

- $\quad$ To examine the relationship between dimensions of Emotional intelligence and mental health among chronic disease group.

- $\quad$ To study the role of Emotional Intelligence in predicting mental health

\section{Hypotheses}

- Different dimensions of Emotional Intelligence would be positively correlated with mental health among chronic disease group.

- $\quad$ Emotional intelligence emerged as predictor of mental health.

\section{Sample}

The sample of the present study consisted of 200 chronic patients of both sexes of age 40 to 60 years with mean age of 48.63 years.

\section{Tools used:}

\section{Multidimensional Measure of Emotional Intelligence (MMEI)}

Multidimensional Measure of Emotional Intelligence (MMEI) scale given by C. R. Darolia (2003) was used. This scale used to provide reliable and valid measurement of emotional intelligence in accordance with Salovey and Mayer's 1990 model. The MMEI is comprised of 80 multiple choice items distributed in five dimensions, each consisting of 16 items. Each item is answered on a five-point scale, viz. very true, mostly true, somewhat true, mostly false, and very false. The sub-dimensions are listed as follows: self-awareness, managing emotions, motivatingoneself, empathy and handling relationships

\section{General Health Questionnaire (GHQ)}

General health questionnaire (GHQ-30) prepared by David Goldberg and Paul Williams (1988) was used to measure general mental health. GHQ-30 is a shorter version of the complete scale. Since the scale was designed to be useful in consulting settings, it focuses on breaks in normal function, rather than upon lifelong traits.

(C) The International Journal of Indian Psychology, ISSN 2348-5396 (e)| ISSN: 2349-3429 (p) | 45 


\section{Emotional Intelligence as Predictor of Mental Health among Chronic Disease Group}

\section{Procedure}

The present study was conducted to examine the emotional intelligence as predictor of mental health of chronic disease persons. To achieve the objective of the study a sample comprised of 200 patients of chronic disease group which was collected from different hospitals of Haryana. Multidimensional Measure of Emotional Intelligence (MMEI) (Darolia, 2003 General Health Questionnaire (GHQ-30) (Goldberg \& Williams, 1988) were administered in different sessions according to the convenience of patients. For data collection, all the participants were individually and personally contacted on their respective places. Major data of chronic disease group was collected from PGI Rohtak Haryana.

\section{Statistical Analysis}

The data collected was rendered to the following statistical analysis:-

a) Pearson product moment method of correlation.

b) Multiple regression analysis (step-wise).

Inter-Correlation Matrix of Chronic Disease Group (N-200)

\begin{tabular}{|l|l|l|l|l|l|l|}
\hline VARIABLE & SA & ME & MO & E & HR & MH \\
\hline SA & 1 & & & & & \\
\hline ME & $.42^{* *}$ & 1 & & & & \\
\hline MO & $.22^{* *}$ & $.45^{* *}$ & 1 & & & \\
\hline E & .09 & .02 & .06 & 1 & & \\
\hline HR & $.17^{*}$ & $.42^{* *}$ & $.52^{* *}$ & .02 & 1 & \\
\hline MH & .04 & $-.27^{* *}$ & $-.44^{* *}$ & $.21^{* *}$ & $-.55^{* *}$ & 1 \\
\hline
\end{tabular}

The findings mentioned in Table1 (Inter-correlation matrix) describe the relationship between emotional intelligence and mental health among chronic disease group. The Table reveals that the measure of mental health is significantly correlated with four factors of emotional intelligence out of total five factors. Self-awareness, the first measure of emotional intelligence has no significant correlation with the measure of mental health for the total sample of chronic disease group.

Managing emotions, the second measure of emotional intelligence is significantly negatively correlated with the measure of mental health $(\mathrm{r}=-.27, \mathrm{p}<.01)$ (Table 1).

The third measure of emotional intelligence motivating oneself $(r=-.44, p<.01)$ is significantly negatively correlated with the measure of mental health (Table 1).

Empathy, the fourth measure of emotional intelligence is positively correlated with the measure of mental health $(\mathrm{r}=.21, \mathrm{p}<.01)$ (Table 1$)$. 


\section{Emotional Intelligence as Predictor of Mental Health among Chronic Disease Group}

Handling relationships, the fifth measure of emotional intelligence $(r=-.55, p<.01)$ is also significantly negatively correlated with the measure of mental health among chronic disease group (here negative correlation implies the positive relationship due to the reverse scoring procedure of the measure of mental health) (Table 1). It clearly means that managing emotions, motivation oneself and skills of handling relationships has been related with better mental health. The positive correlation between empathy (a dimension of emotional intelligence) and the measure of mental health implies the negative association between them, which means that over sensitivity to other' feelings, concerns, and taking their perspective lead to poor mental health. On the basis of these findings the first hypothesis that states "Different dimensions of emotional intelligence would be positively correlated with mental health among chronic disease group” got accepted.

\section{Regression Analysis}

Regression analysis was conducted to identify the specific determinants of mental health of chronic disease group . For this purpose method of stepwise multiple regression was used.

Multiple $R$ and $\boldsymbol{R}^{2}$ change for mental health in chronic diseases group (as a consequence of step-wise regression equation)

\begin{tabular}{|l|l|l|l|l|l|l|l|}
\hline Predictor & $\mathbf{R}^{2}$ & $\begin{array}{l}\mathbf{R}^{2} \\
\text { Change }\end{array}$ & $\boldsymbol{\beta}$ & $\mathbf{S E}$ & $\mathbf{B}$ & t-value & Sig. \\
\hline Constant & & & 44.84 & 5.91 & & 7.59 & .00 \\
\hline $\begin{array}{l}\text { EI (Handling } \\
\text { relationships) }\end{array}$ & .300 & .300 & -.43 & .08 & -.33 & 5.41 & .00 \\
\hline $\begin{array}{l}\text { EI(Motivating } \\
\text { oneself) }\end{array}$ & .400 & .037 & -.30 & .08 & -.24 & 3.99 & .00 \\
\hline EI (Empathy) & .434 & .034 & .26 & .08 & .17 & 3.18 & .00 \\
\hline
\end{tabular}

Findings mentioned in Table no.2 indicate that the variable of handling relationships (a sub variable of emotional intelligence), motivating oneself, and empathy (sub variables of emotional intelligence), met the criterion to enter in the regression equation and explained $43 \%$ of variance for mental health among the group of chronic disease. It indicated that all these variables are predictors of mental health.

The first variable that is handling relationships (a sub variable of emotional intelligence) entered in regression equation with $\mathrm{R}^{2} 0.300(\mathrm{~F}=85.03, \mathrm{p}<.01)$, and $\mathrm{b}$-value is -0.33 , which is significant at .01 level, which means that handling relationships accounted for $30 \%$ of variance in criterion variable i.e. mental health (Table 2).

The second variable motivating oneself (a sub variable of emotional intelligence) entered in the regression equation with $\mathrm{R}^{2} 0.400(\mathrm{~F}=43.53, \mathrm{p}<.01)$, and $\mathrm{b}$-value is -0.24 , which is significant at .01 level, which implies that motivating oneself accounted for $40 \%$ of variance in the criterion 


\section{Emotional Intelligence as Predictor of Mental Health among Chronic Disease Group}

variable together with handling relationships and realistic acceptance and only $4 \%$ of variance, when taken alone (Table 2).

The results presented in Table 2 further revealed that at the third and last step empathy (a sub variable of emotional intelligence) entered in the regression equation with $R^{2} 0.434(F=37.33$, $\mathrm{p}<.01$ ), and $\mathrm{b}$-value is 0.17 , which is significant at .01 level. This implies that empathy together with other predictors (i.e. handling relationships, realistic acceptance and motivating oneself) explains $43 \%$ of variance in the criterion variable, whereas at its own level it contributes only $3 \%$ of variance.

Tsaousis and Nikolaou (2005) also revealed that there is a relationship between emotional intelligence and health functioning and hierarchical regression analysis indicated the unique contribution of each of the emotional intelligence scales on the overall health score. Choubey et al. (2009) revealed that emotional intelligence and its various component abilities are associated with better health outcomes and lower levels of stress. Among the dimensions of emotional intelligence, the ability to manage emotion in self was found the best predictor of stress as well as health and regression analyses identified emotional intelligence as a positive resource in high stress condition.

\section{Acknowledgments}

The author appreciates all those who participated in the study and helped to facilitate the research process.

\section{Conflict of Interests}

The author declared no conflict of interests.

\section{REFERENCES}

Booth-Kewley, S., \& Friedman, H. S. (1987). Psychological predictors of heart disease: A quantitative review. Psychological Bulletin, 101, 343-362. http://journals.lww.com/ijgc/Abstract/2005/09000/The_contribution_of_spirituality_and_ spiritual.7.aspx

Braam, A.W., Hein, E., Deeg, D. J., Twisk, J. W., Beekman, A. T., Van Tilburg, W. (2004). Religious involvement and 6-year course of depressive symptoms in older Dutch citizens: results from the Longitudinal Aging Study Amsterdam. Journal of Aging and Health, 16(4), 467-489. http://jah.sagepub.com/content/16/4/467.full.pdf

Brown, R. F., \& Schutte, N. S. (2006). Direct and indirect relationships between emotional intelligence and subjective fatigue in university students. Journal of Psychosomatic Research, 60, 585-593. 


\section{Emotional Intelligence as Predictor of Mental Health among Chronic Disease Group}

Ciarrochi, J., Deane, F. P., \& Anderson, S. (2002). Emotional intelligence moderates the relationship between stress and mental health. Journal of Personality and individual differences, 32(2), 197-209.

Choubey, A. K., Singh, S. K., \& Pandey, R. (2009). Role of Emotional Intelligence in Stress and Health. Indian Journal of Social Science Researches, 6(1), 122-134. http://ijssr.110mb.com/IJSSR-March-2009-vol-6.pdf\#page=125

Goleman, D., (1995). Emotional Intelligence, New York: A Bantam Book.

Matthews, G. \& Zeidner, M. (2000). Emotional intelligence, adaptation to stressful encounters, and health outcomes. In R. Bar-On, \& J.D.A. Parker (Eds), The handbook of emotional intelligence. San Francisco, CA: Jossey-Bass

Mayer, J., \& Salovey, P. (1997). What is Emotional Intelligence? In P. Salovery and D. J .Shyter (Eds,), Emotional Development and Emotional Intelligence, New York: Basic Books.

Schutte, N. S., Malouff, J. M., Bobik, C., Conston, T., Greeson, C., Jedlicka, C., et al. (2001). Emotional intelligence and interpersonal relations. Journal of Social Psychology, 141(4), 523-536.

Salovey, P., Mayer, J. D., Goldman, S., Turvey, C., \& Palfai, T. (1995). Emotional attention, clarity, and repair: exploring emotional intelligence using the trait meta-mood scale. In J. W. Pennebaker (Ed.), Emotion, disclosure, and health (pp. 125-154). Washington, DC: American Psychological Association.

Taylor, G. J. (2001). Low emotional intelligence and mental illness. In J. Ciarrochi, \& J.P. Forgas (Eds), Emotional intelligence in everyday life: A scientific enquiry (pp. 67-81). Philadelphia, PA: Taylor \& Francis

Tsaousis, I., \& Nikolaou, I. (2005). Exploring the relationship of emotional intelligence with physical and psychological heath functioning. Journal of Stress and Health, 21(2), 77-86.

How to cite this article: Manju (2016), Emotional Intelligence as Predictor of Mental Health among Chronic Disease Group, International Journal of Indian Psychology, Volume 3, Issue 4, No. 58, ISSN 2348-5396 (e), ISSN: 2349-3429 (p), DIP: 18.01.043/20160304, ISBN: 978-1-36524976-1 